International Journal of Modern Physics D

(C) World Scientific Publishing Company

\title{
SEARCH FOR THE STANDARD MODEL HIGGS BOSON WITH THE ATLAS DETECTOR
}

\author{
Domizia Orestano \\ On behalf of the ATLAS Collaboration \\ Dipartimento di Matematica e Fisica, Università degli Studi di Roma Tre, \\ Istituto Nazionale di Fisica Nucleare, Sezione di Roma Tre, via della Vasca Navale 84 \\ Roma, 00146, Italy \\ orestano@fis.uniroma3.it
}

Received 3 March 2013

\begin{abstract}
This document presents a brief overview of some of the experimental techniques employed by the ATLAS experiment at the CERN Large Hadron Collider in the search for the Higgs boson predicted by the standard model of particle physics. The data and the statistical analyses which allowed in July 2012, only few days before this presentation at the Marcel Grossman Meeting, to firmly establish the observation of a new particle, are described. The additional studies needed to check the consistency between the newly discovered particle and the Higgs boson are also discussed.

Keywords: Higgs, boson, LHC, ATLAS.

PACS numbers:
\end{abstract}

\section{Introduction}

The Large Hadron Collider ${ }^{1}$ (LHC) at CERN provides through the head-on collisions of two counter-rotating proton beams the highest center of mass energy ever achieved in a laboratory. This energy, which increased from $7 \mathrm{TeV}$ in 2011 operation to $8 \mathrm{TeV}$ in 2012, is partially converted in mass of the interaction products and can materialize in particles with masses up to three order of magnitude higher than the proton mass. One of the main goals of the LHC physics program is to search for new particles and in particular to either observe the Higgs boson predicted by the standard model of elementary particles (SM) and measure its properties or disprove its existence. The production of this particle is expected to be very rare with respect to a large variety of concurrent processes, as quantified by a property of the physics processes called cross-section. However the LHC high intensity beams and well tuned optics provide a high luminosity, a machine parameter related to the number of interactions produced per unit of cross-section, and hence a large enough sample of events to be scrutinized could be collected over 2011 and the first part of 2012 data takings. The challenge of resolving the rare signal of the Higgs boson among a huge background of similar particles produced by the energetic collisions is 
achieved through two dedicated detectors, ATLAS ${ }^{2}$ and $\mathrm{CMS}^{3}$. This presentation focuses on the techniques adopted by the ATLAS experiment.

In the next section the SM Higgs boson characteristics will be briefly presented, while section 3 will be devoted to the LHC machine operations and to an overview of the ATLAS detector. The search strategy adopted by ATLAS is described in section 4, focusing in particular on the two most sensitive Higgs boson's decay channels. Finally in section 5 all the results available from ATLAS at the time of this communication are combined together providing a clear evidence for a new particle. The interpretation of this result and the perspectives for the study of this new signal at the LHC are briefly discussed in the conclusions.

\section{The SM Higgs boson}

The SM of particle physics, proposed in the $60 \mathrm{~s}^{4-7}$, describes the fundamental particles and the electromagnetic, weak, and strong forces between them, providing so far a very successful description of the available experimental data. In the SM, forces are mediated by the exchange of particles with integer spin, which are known as vector bosons. These bosons are exchanged between electromagnetic, weak, and strong charges. The charges are carried by the fundamental constituents of matter, six quarks and six leptons, and their antiparticles. These are all of semi-integer spin and are known as fermions. The photon $(\gamma)$, the boson that mediates electromagnetism, and gluons, the bosons that mediate the strong force, are massless. The carriers of the weak force, the $W$ and $Z$ bosons, have instead masses $\sim 100$ times that of the proton. In the SM, the $W$ and $Z$ bosons obtain their mass through their interactions with a field of weak charge that is postulated to pervade the vacuum ${ }^{8-10}$ of space. Excitation of this field would produce a massive particle with zero spin: the Higgs boson $(H)$.

The SM is unable to provide any direct indication of the mass $\left(m_{H}\right)$ of the postulated particle, an information critical for the experimentalists to design the appropriate search strategies. It is not only the center of mass energy required to produce the Higgs boson which varies with its mass but most of the associated phenomenology. Indeed the model predicts that the coupling of the Higgs boson to other particles depends both on its mass and on the mass of the coupled particles. In particular the coupling to the fermions scales as the fermion mass while the coupling to the force mediating bosons scales as the square of the vector boson mass. From these dependencies most of the SM Higgs boson phenomenology can be derived. For instance whenever $m_{H}$ exceeds the threshold for the decay in a "more massive" final state the latter becomes dominant over the ones available at a lower mass and the relative decay widths (branching ratios, BR), are all affected. This effect is particularly striking when the decay to a pair of on-shell (real) $W$ bosons becomes accessible. Moreover the Higgs boson total decay width varies strongly with $m_{H}$, going from few $\mathrm{MeV}$ at $m_{H}=100 \mathrm{GeV}$ up to $1 \mathrm{TeV}$ at $1 \mathrm{TeV}$, where the same concept of particle as a well defined energy state breaks down completely. 
The search for the SM Higgs boson was performed over various decades at lower center of mass energies and its non-observation allowed to set limits on $m_{H}$, in particular the ones provided by the CERN Large Electron Positron collider (LEP), of $114.4 \mathrm{GeV}$ at the $95 \% \mathrm{CL}^{11}$ and by the Tevatron proton-antiproton collider at Fermilab, excluding the mass region 147 to $180 \mathrm{GeV}$ at the $95 \% \mathrm{CL}^{12}$. Indirect indications for a "low" value mass, close to $100 \mathrm{GeV}$ (as opposed to values up to $\sim 500 \mathrm{GeV}$ ), have been provided by the analysis ${ }^{13}$ of precision measurements of properties of known particles to which the Higgs boson mass contributes through quantum mechanical effects. The mass range left for the LHC to explore is vast and the possible scenarios, both in terms of signal abundance and favored decay channels, vary rapidly across it. For instance the cross-section for the production of a SM Higgs boson, dominated by the gluon-gluon fusion process, at $\sqrt{s}=7 \mathrm{TeV}$ changes from $24 \mathrm{pb}$ if $m_{H}=100 \mathrm{GeV}$ to $1 \mathrm{pb}$ if $m_{H}=500 \mathrm{GeV}$. Focusing on a specific decay channel, like $H \rightarrow Z Z^{*}$, the BR would change from $0.1 \%$ to $26 \%$ and the event rate, going as $\sigma \times B R$, would globally increase, while for other channels, like $H \rightarrow \gamma \gamma$ the rate would go through a maximum and then drop with $m_{H}{ }^{14}$.

This variety of decay channels, production rate and decay width, imposes an experimental approach as inclusive as possible: given a machine providing the highest possible energy and highest possible intensity the detector systems should be able to scan the full mass range under investigation exploiting the signature of the Higgs boson decay in all the achievable decay channels, and this is what the LHC project and the ATLAS and CMS experiments have done.

\section{The ATLAS experiment at the LHC}

The design of the ATLAS detector ${ }^{2}$ was optimized to study a broad range of physics processes, including SM Higgs boson production, over a wide mass range. The entire detector is $44 \mathrm{~m}$ long and $25 \mathrm{~m}$ in diameter. It is located in an underground cavern at a depth of $100 \mathrm{~m}$, where it surrounds one of the collision points around the 27-km-long LHC ring. ATLAS is composed of several distinct subdetectors, organized in concentric cylindrical layers, which provide a response to the various types of particles emerging from the interaction, allowing to identify them and to measure their energy and momentum, thus reconstructing the dynamics of the collision. The momenta of charged particles are measured by an inner tracking detector (ID) immersed in a $2 \mathrm{~T}$ axial field provided by a solenoidal superconducting magnet. The energies of electrons and photons are measured in an electromagnetic calorimeter (ECAL) that surrounds the inner detector and magnet. An additional layer of calorimeters outside the ECAL for measuring hadrons (such as protons and neutrons) also serves as an absorber, so that only energetic muons and the elusive weakly interacting neutrinos penetrate it. The muon spectrometer surrounds the calorimeters; it consists of superconducting magnets providing a toroidal field and a system of precision charged-particle detectors.

The combination of the subdetectors provides particle energy and momentum 
measurements, together with electron, muon, and photon identification, over more than $98 \%$ of the solid angle. The measurements are made by $\sim 90$ million sensor elements, most of which are in the inner detector. Jets (narrow cones of particles produced by the conversion of quarks and gluons to hadrons) are reconstructed by using the nearly $4 \pi$ solid angle coverage of the calorimeters. In high-energy proton-proton collisions, only one constituent (a quark or a gluon) from each proton takes part in the interactions that result in a wide variety of known and possibly unknown processes, including production of the SM Higgs boson. The remnants of two colliding protons tend to travel along the beam directions and exit the detector unobserved, so it is only possible to study momentum balance in the plane transverse to the proton beam axis. Neutrinos, which are normally not directly detectable, are inferred from their transverse momenta; they are assumed to balance the sum of the transverse momenta $\left(p_{\mathrm{T}}\right)$ of the observed electrons, muons, photons, and jets. Their presence is thus indicated by the magnitude of the missing transverse momentum, denoted by $E_{\mathrm{T}}^{\mathrm{miss}}$.

During standard LHC operation, two counter-rotating packets of protons cross at the center of ATLAS every $50 \mathrm{~ns}$. The high intensity of the proton beams results in multiple proton-proton collisions occurring during each crossing of proton packets. The average number of interactions per proton-packet (bunch) crossing was about 10 in 2011, and it doubled in the first part of 2012. This effect, known as pile-up, is particularly challenging from the experimental point of view. Although the chances of more than one interesting interaction occurring in a bunch-crossing are negligible, the underlying interactions perturb the measurement of the kinematic variables for the interaction of interest, deteriorating the resolution. Particularly sensitive are the energy deposited in the calorimeters and the $E_{\mathrm{T}}^{\mathrm{miss}}$, which being measured indirectly, inherits from all the alterations in the direct measurements. The challenge consists in building observable quantities, out of the raw measurements, which once properly calibrated do not display a dependence upon the number of interactions per bunchcrossing.

The analyses discussed here are based on the data collected in the 2011 run, when LHC operated at a center of mass energy of $7 \mathrm{TeV}$, delivering to ATLAS an integrated luminosity ${ }^{\mathrm{a}}$ of approximately $5.6 \mathrm{fb}^{-1}$, and on the data collected in the period April to June 2012, when the center of mass energy was raised to $8 \mathrm{TeV}$ and the integrated luminosity was approximately $6.3 \mathrm{fb}^{-1}$. Typical analysis selections, asking for all the detectors being operational, reduce the useful statistics to approximately 4.7 and $5.8 \mathrm{fb}^{-1}$ in the two data periods. The analysis starts already on-line during the data taking, defining through a rapid three-level decision taking system, called trigger, the bunch-crossings for which the data need to be kept for further analysis. The trigger system reduces the rate from $20 \mathrm{MHz}$ down

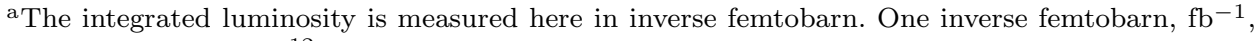
corresponds to $60 \times 10^{12}$ interactions, out of which the experiments need to extract few interesting signal events. 
to few hundred $\mathrm{Hz}$, requesting the presence of high transverse momentum objects in the final state (muons, electrons, photons, jets...) or of high missing transverse momentum. Thresholds are kept as low as possible to reduce the bias on the selected events. Typical trigger thresholds at the last trigger level are $24 \mathrm{GeV}$ transverse momentum for single isolated electrons and muons, 8-18 GeV for di-leptons, 20$35 \mathrm{GeV}$ for di-photons, $20-29 \mathrm{GeV}$ for di-taus and $80 \mathrm{GeV}$ for missing transverse momentum.

\section{The search for the SM Higgs boson in ATLAS}

At the time of this presentation the search for the SM Higgs boson has been performed over the full statistics collected in 2011 for most of the accessible final states and is still ongoing for the 2012 data collected up to June. Table 1 summarizes the analyses performed on 2011 data, and includes references to each study.

Table 1. Summary of ATLAS SM Higgs searches performed with the 2011 data, showing the decay channel, mass range and analysis references. Multiple mass ranges indicate points where the analysis changes significantly.

\begin{tabular}{ccc}
\hline Channel & $m_{H}$ range $(\mathrm{GeV})$ & Reference \\
\hline$H \rightarrow \gamma \gamma$ & $110-150$ & 15 \\
$H \rightarrow Z Z^{*} \rightarrow 4 \ell$ & $110-600$ & 16 \\
$H \rightarrow Z Z \rightarrow \ell^{+} \ell^{-} q \bar{q}$ & $200-280-600$ & 17 \\
$H \rightarrow Z Z \rightarrow \ell^{+} \ell^{-} \nu \bar{\nu}$ & $200-300-600$ & 18 \\
$H \rightarrow W W^{*} \rightarrow \ell^{+} \nu \ell^{-} \bar{\nu}$ & $110-200-300-600$ & 19 \\
$H \rightarrow W W \rightarrow \ell \nu q \bar{q}$ & $300-600$ & 20 \\
$H \rightarrow \tau^{+} \tau^{-} \rightarrow \ell^{+} \ell^{-}, \ell \tau_{h}, \tau_{h} \tau_{h}$ & $110-150$ & 21 \\
$W H \rightarrow \ell \nu b \bar{b} ; Z H \rightarrow \ell^{+} \ell^{-} b \bar{b}, \nu \bar{\nu} b \bar{b}$ & $110-130$ & 22 \\
$W H \rightarrow W W W \rightarrow \ell \nu \ell \nu \ell \nu$ & $110-300$ & 23 \\
\hline
\end{tabular}

Out of the combination of most of these analyses ${ }^{24}$, a first important result is achieved. The presence of a SM Higgs boson is excluded at 95\% CL over most of the search range, in particular the Higgs boson mass ranges of $111.4 \mathrm{GeV}$ to 116.6 GeV, $119.4 \mathrm{GeV}$ to $122.1 \mathrm{GeV}$, and $129.2 \mathrm{GeV}$ to $541 \mathrm{GeV}$ are excluded at the $95 \%$ confidence level, while the range $120 \mathrm{GeV}$ to $560 \mathrm{GeV}$ is expected to be excluded in the absence of a signal. An excess of events is observed at Higgs boson mass hypotheses around $126 \mathrm{GeV}$ with a local significance of 2.9 standard deviations (sigma). The global probability for the background to produce an excess at least as significant anywhere in the entire explored Higgs boson mass range of 110-600 GeV is estimated to be $\sim 15 \%$, corresponding to a significance of approximately one sigma. The excess is seen in the $\gamma \gamma$ and in the $Z Z^{*}$ to four charged leptons final states, which are expected to be the most sensitive channels in that mass region and the channels with the best $m_{H}$ resolution. It must be mentioned that for masses $\sim 125 \mathrm{GeV}$ the SM Higgs boson mass peak is expected to be very narrow and the 
width of the experimentally reconstructed mass distribution should be dominated by the detector resolution. In order to illustrate some of the techniques adopted in the analysis this summary will focus on these same two channels, for which the results on the first part of 2012 are now available, and for which the 2011 data have also been re-analyzed after analysis re-optimization.

\subsection{Search for the SM Higgs decay into four charged leptons through $Z Z^{*}$}

The decay of the SM Higgs into two $Z$ bosons, which subsequently decay into two pairs of oppositely charged same flavor leptons has always been considered as the golden decay channel for the Higgs at the LHC for its high signal over background ratio. High $p_{\mathrm{T}}$ muons and electrons, tau leptons are not explicitly considered here, are a clean signature of interesting events in the crowded environment generated by the high intensities and high energies of the LHC p-p collisions. Four leptons have a negligible probability to be accidentally found in the same event. Moreover this decay does not contain undetected particles and the mass of the Higgs boson is completely reconstructed from the kinematics of the four final state leptons. For Higgs boson masses above twice the mass of the $Z$ boson both the $Z$ s produced from the $H$ decays would have to be real and the analysis could benefit from the additional constraint of two pairs of leptons with invariant masses compatible to the $Z$ mass. This is not the case for a Higgs boson with mass below $180 \mathrm{GeV}$, which would then decay in at least one virtual (off-shell) $Z$, denoted as $Z^{*}$, whose mass is lower than the mass of the real (on-shell) $Z$ boson.

In this channel the Higgs boson signal appears as a narrow peak in the fourlepton invariant mass distribution over a background dominated by continuum $Z Z^{*}$
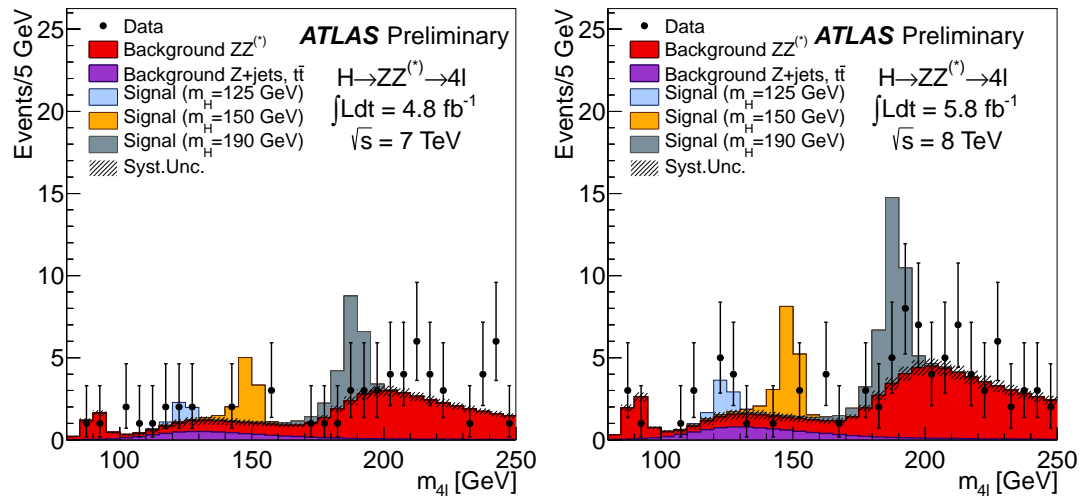

Fig. 1. The distribution of the four-lepton invariant mass, $m_{4 \ell}$, for the selected candidates compared to the background expectation in the $80-250 \mathrm{GeV}$ mass range for the (left) $\sqrt{s}=7 \mathrm{TeV}$ and (right) $\sqrt{s}=8 \mathrm{TeV}$ datasets. Error bars represent $68.3 \%$ central confidence intervals. The signal expectation for several $m_{H}$ hypotheses is also shown ${ }^{26}$. 
production. The small branching ratios involved mean that only few candidates are expected and it is critical to keep a high efficiency for the identification and measurement of low $p_{\mathrm{T}}$ leptons. Before analyzing the 2012 data, the kinematic selections were re-optimized to increase the sensitivity at low $m_{H}$. For 2012 data, improved electron reconstruction and identification algorithms were used, allowing for the effects of electron bremsstrahlung in the track pattern recognition, improving the stability against pileup and refitting the final tracks with a Gaussian Sum Filter algorithm ${ }^{25}$ to account for energy losses in the track fit; the last improvement was also applied in a re-analysis of 2011 data. Small improvements were also made to increase the muon reconstruction coverage. The kinematic selections require four leptons with minimum $p_{\mathrm{T}}$ of at least $6 \mathrm{GeV}$. Additional selections require that the invariant masses of leading $\left(m_{12}\right)$ and sub-leading $\left(m_{34}\right)$ lepton pairs lie within ranges compatible with an on-shell $Z$ decay and the decay of a virtual $Z$ whose mass would increase with the hypothesis on $m_{H}$, respectively. The reconstruction and selection efficiency was improved, for a hypothesized value of $m_{H}=130 \mathrm{GeV}$, from $27 \%$ for the old 2011 analysis to $41 \%$ for the 2012 in the four muon final state, and from $14 \%$ to $23 \%$ in the four electrons final state.

The spectrum of the four-lepton invariant mass, $m_{4 \ell}$, is compared to background expectations from simulation carefully normalized to data using control regions, in Fig. 1-left and 1-right, for 2011 and 2012 data respectively. An excess of events compatible with SM Higgs boson production is visible around $125 \mathrm{GeV}$, particularly in the $4 \mu$ and $2 \mathrm{e} 2 \mu$ channels, for which in the mass window $120-130 \mathrm{GeV} 6$ and 5 candidate events are observed respectively, adding 2011 and 2012 data, against a background expectation of $1.30 \pm 0.08$ and $2.19 \pm 0.21$. The expected number of signal events in the same mass interval is $2.09 \pm 0.28$ in $4 \mu$ and $2.28 \pm 0.31$ in $2 \mathrm{e} 2 \mu$. More details on this analysis can be found in Ref. 26.

\subsection{Search for the SM Higgs decay into $\gamma \gamma$}

The Higgs boson signal in the $H \rightarrow \gamma \gamma$ channel is expected to appear as a narrow peak in the invariant mass distribution $m_{\gamma \gamma}$ of the two photons. There are many sources of photons, real or fake, which could mimic the same final state but they are not expected to present any resonant behavior, they should rather produce a slowly varying $m_{\gamma \gamma}$ background distribution. The background level and shape can be fitted from the data. Data are divided into several analysis categories according to their different invariant mass resolution and signal/background ratios, associated to different properties of the selected photons in each event. The background comes mainly from irreducible continuum $\gamma \gamma$ production and $\gamma$-jet production with one jet misidentified as an isolated photon, with a smaller contribution from jet-jet events. The analysis re-optimization for this channel lead to a better photon identification algorithm being deployed for 2011 data and to the application of a higher $p_{\mathrm{T}}$ requirement (30 instead of $25 \mathrm{GeV}$ ) on the second photon, while keeping the threshold for the first one at $40 \mathrm{GeV}$. For 2012 data, a photon identification, optimized for 
stable performance with high pileup, was used. Besides these improvements in the objects definition the analysis was modified to explicitly include a new category of events related to the production of the Higgs boson through the Vector Boson Fusion (VBF) mechanism instead of the dominant gluon-gluon fusion. The VBF mechanism foresees the fusion to a Higgs boson of the two $W$ bosons radiated from a quark and an anti-quark in the initial state. The two quarks, after radiating the $W \mathrm{~s}$, are expected to produce two forward jets. The new category contains therefore the events with at least two high- $p_{\mathrm{T}}$ jets separated by at least 2.8 units in pseudorapidity and having invariant mass above $400 \mathrm{GeV}$, in addition to the two photon candidates. The remaining events were classified into nine separate categories, depending on whether the photons were reconstructed as converted or unconverted candidates, their positions within the calorimeter, and the transverse momentum of the di-photon system with respect to the event thrust axis.

To reconstruct the $\gamma \gamma$ invariant mass both the energy and the angles of the reconstructed photons are needed. The angles were determined from the vertex reconstruction, obtained both using the longitudinal segmentation in the liquid-argon electromagnetic calorimeter, and the reconstructed photon conversion vertex (where available). A resolution of about $15 \mathrm{~mm}$ along the beams direction was achieved for the vertex. This information was combined with primary vertex information from the inner detector tracking to associate jets to the correct primary vertex in the 2 -jet category.

The $m_{\gamma \gamma}$ distribution in each category was fitted using the sum of a signal and a background model. The expected invariant mass spectrum for $H$ decays was modeled using a combination of Gaussian and Crystal Ball functions, where the full-width half-maximum resolution varies from 3 to $6 \mathrm{GeV}$ depending on the event category, while the background could be described by different shapes amongst 4 th-order Bernstein polynomials, exponentials of 2nd-order polynomials or a simple

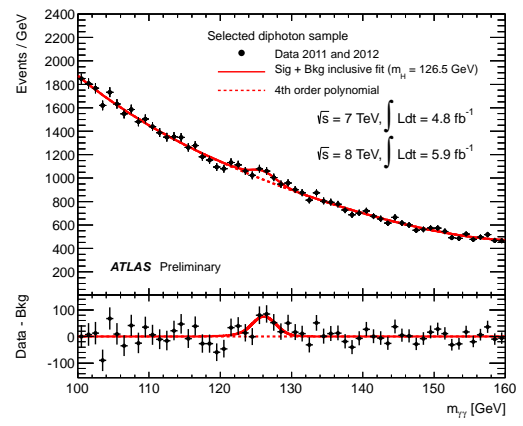

Fig. 2. Invariant mass distribution for the combined $\sqrt{s}=7 \mathrm{TeV}$ and $\sqrt{s}=8 \mathrm{TeV}$ data samples. Superimposed is the result of a fit including a signal component fixed to a hypothesized mass of 126.5 GeV and a background component described by a fourth-order Bernstein polynomial. The bottom inset displays the residual of the data with respect to the fitted background ${ }^{27}$. 
exponential. The choice of the background model was based on extensive studies of high-statistics simulated samples of $\gamma \gamma, \gamma$-jet and jet-jet events, taking into account the potential bias and fit stability given the expected data statistics in each category. The residual biases on a potential $125 \mathrm{GeV}$ signal seen in background-only simulation studies were taken as systematics on the fitted signal yield in data.

The inclusive $m_{\gamma \gamma}$ distribution combining the 23788 selected events from the 2011 data and 35271 events from the 2012 data, before separation into the ten analysis categories, is shown in Fig. 2. A fit to a 4th-order Bernstein polynomial background and a signal component at $126.5 \mathrm{GeV}$ shows a significant excess at this mass. However, the full analysis results are obtained by fitting the $m_{\gamma \gamma}$ distributions of the ten categories separately with a common hypothesis for the signal strength $\mu$, the ratio between the observed cross-section and the SM one. More details can be found in Ref. 27.

\section{Combination of the results from the different ATLAS searches}

The two searches described in the last section have both given results compatible with the presence of a SM Higgs signal. These results can be interpreted for each analysis separately and give a 3.4 sigma excess with respect to the background hypothesis at $m_{H} \sim 125 \mathrm{GeV}$ in $Z Z^{*}$ and 4.5 sigma at $m_{H} \sim 126.5 \mathrm{GeV}$ in $\gamma \gamma$. It is however far more interesting to combine them together and with all the other results, both to improve the statistical significance and to establish if they are compatible with each other. Indeed the SM imposes correlations between the results of the different searches. If there is only one Higgs boson, the SM one, the only ingredient not provided by the model is the mass $m_{H}$, which should of course be the same within the experimental uncertainties when the signal is observed through different decay channels. Cross-sections and branching ratios for instance are well defined once $m_{H}$ is known, therefore the model can predict the intensity of the signal
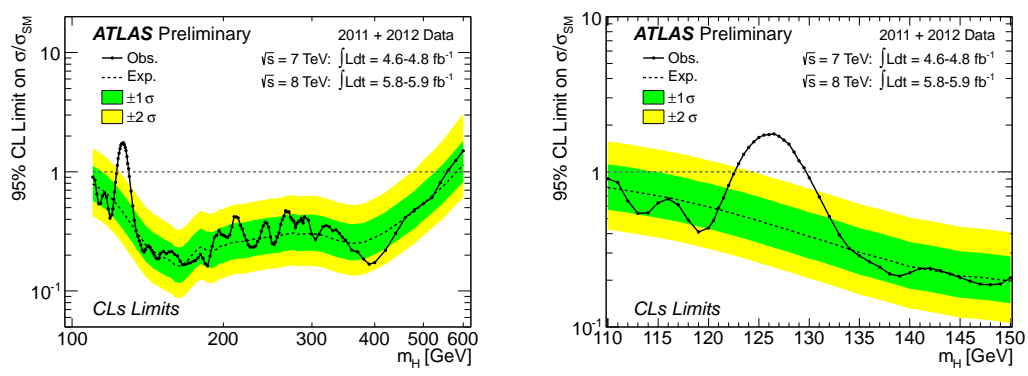

Fig. 3. The observed (full line) and expected (dashed line) 95\% CL combined upper limits on the SM Higgs boson production cross section divided by the SM expectation as a function of $m_{H}$ in the full mass range considered in this analysis ${ }^{29}$. The dashed curves show the median expected limit in the absence of a signal and the green and yellow bands indicate the corresponding $68 \%$ and $95 \%$ intervals. 
to be observed in each channel. Assuming SM branching ratios results are often presented in terms of the signal strength $\mu=\sigma / \sigma_{\mathrm{SM}}$. Standardized prescriptions ${ }^{28}$ are adopted in presenting and combining results in order to allow experiment-toexperiment comparisons and subsequent combination of ATLAS and CMS results.

In order to combine the outcome of the searches performed in different channels a dedicated likelihood-based procedure, which takes into account signal and control regions from each analysis and correlations between the analyses ${ }^{24}$, is adopted. The compatibility with or exclusion of a particular SM Higgs mass hypothesis are tested by scaling the predicted Higgs boson contribution in the likelihood fit by a signal the strength parameter $\mu$, applied coherently to all channels. The exclusion of $\mu=1$ at a particular Higgs mass hypothesis corresponds to the exclusion of the production of a SM Higgs boson at that mass. The $95 \%$ confidence level exclusion limits obtained with the complete 2011 dataset, plus the 2012 first data for the $\gamma \gamma$ and $Z Z^{*}$ channels $^{29}$, can be derived from the plot in Fig. 3. The experiment is sensitive to a Higgs boson signal in the mass range within which the expected limit lies below $\mu=1$. If in that region the observed limit lies within the $95 \%$ band around the expected limit then a Higgs boson of that mass can be excluded. This is the case for masses in the range $110 \mathrm{GeV}$ to $122.6 \mathrm{GeV}$ and $129.7 \mathrm{GeV}$ to $558 \mathrm{GeV}$. An excess of events is observed for a Higgs boson mass hypothesis near $126.5 \mathrm{GeV}$.

In order to quantify the significance of the excess, the compatibility with the background-only hypothesis is evaluated. The local $p_{0}$ in Fig. 4 represents the probability that the background would fluctuate to produce the number of events observed in data in absence of a signal for a particular value of $m_{H}$. The most significant deviation (smallest local $p_{0}$ ) corresponds to 5.1 sigma, at $m_{H}=126.5 \mathrm{GeV}$, close to the expected significance for a SM Higgs boson at this mass (4.6 sigma). The excess is mildly sensitive to systematic uncertainties on the energy scale and resolution for
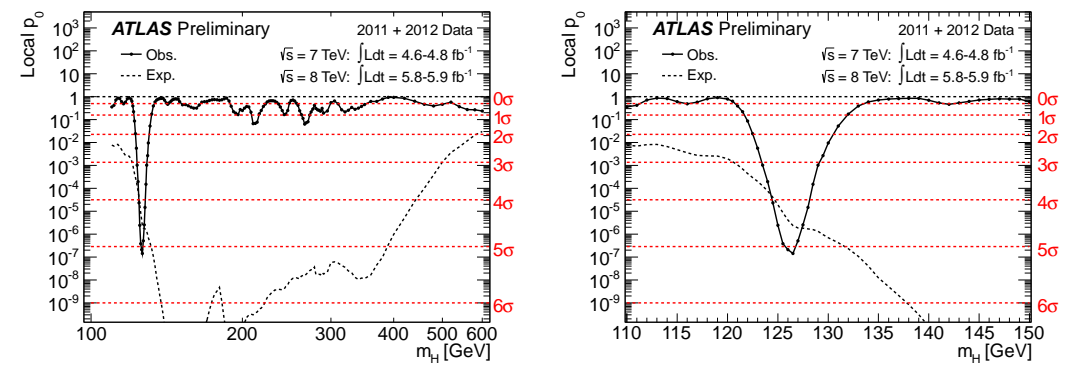

Fig. 4. The local significance in the low mass range of this analysis as a function of $m_{H}{ }^{29}$. An excess (deficit) of events corresponds to a positive (negative) local significance. The dashed curves show the median expected local $p_{0}$ under the hypothesis of a SM Higgs boson production signal at that mass. The horizontal dashed lines indicate significances ranging from 0 to 6 sigma. Energy scale systematics are not included; taking them into account leads to a small correction of about 0.1 sigma near $m_{H}=126 \mathrm{GeV}$. 
photons and electrons, which reduce the significance to 5.0 sigma. However the small probability of a local background fluctuation may lead to a non negligible probability when the explored mass range is large. This effect, known as look-elsewhere effect, has to be taken into account. The evaluation for the entire search mass range from 110 to $600 \mathrm{GeV}$ gives a global significance of 4.1 sigma.

The evidence for a new particle is achieved combining all the search channels. The SM imposes relations between the different searches. Are they verified? Once established that there is a signal the strength parameter $\mu$ can be extracted separately for the different channels. Fig. 5 compares the signal strengths measured in the different channels to the expected SM value $\mu=1$. Within the still large experimental uncertainties all the channels appear to be compatible with the SM expectation. A more stringent compatibility test can be performed by minimizing the likelihood with respect to both $\mu$ and $m_{H}$ in each channel and comparing the results in a two-dimensional plane. An example, including only the two channels

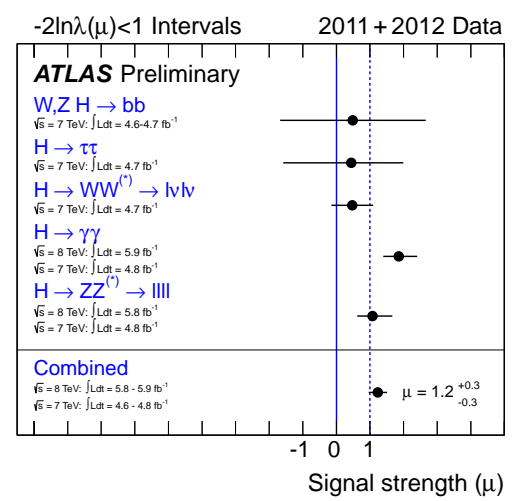

Fig. 5. Summary of the individual and combined best-fit values of the strength parameter ${ }^{29}$ for a Higgs boson mass hypothesis of $126.5 \mathrm{GeV}$.

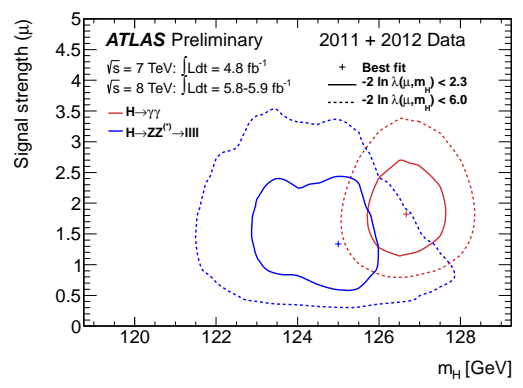

Fig. 6. Likelihood contours in $\left(\mu, m_{H}\right)$ for the $H \rightarrow Z Z^{*} \rightarrow 4 \ell$ and $H \rightarrow \gamma \gamma$ channels $^{29}$ including energy scale systematics . 
with better mass resolution, is given in Fig.6.

\section{Conclusions}

This contribution summarizes the observation by the ATLAS collaboration of a new particle. A more extensive description of this result can be found in Ref.30 and in Ref.31. At the same time and independently from ATLAS, the same conclusion has been reached by the analysis over a similar dataset and with similar statistical significance, by the CMS collaboration ${ }^{32}$. While there is no doubt that a new object has been produced and observed through its decays in $\gamma \gamma$ and $Z Z^{*}$, a clear evidence for its decay also in other channels expected for a SM Higgs boson of $m_{H} \sim 125 \mathrm{GeV}$ in other channels is still pursued. The observation in all the expected channels, with the expected strength, is indeed essential to prove, or disprove, the identification of the new object with the SM Higgs boson. A mass of $\sim 125 \mathrm{GeV}$ is particularly favorable from his point of view since for this value many decay channels are accessible to LHC experimentalists, who will provide many constraints to bound the model parameters.

\section{References}

1. L. Evans, P. Bryant, Eds., JINST 3, S08001 (2008).

2. ATLAS Collaboration, JINST 3, S08003 (2008).

3. CMS Collaboration, JINST 3, S08004 (2008).

4. S.L. Glashow, Nucl. Phys. 22 (1961) 579.

5. S. Weinberg, Phys. Rev. Lett. 19 (1967) 1264.

6. A. Salam, in Elementary Particle Physics, (Almquvist and Wiksell, Stockholm, 1968) pp. 367 .

7. G. 't Hooft, M. Veltman, Nucl. Phys. B 44 (1972) 189.

8. F. Englert, R. Brout, Phys. Rev. Lett. 13 (1964) 321.

9. P.W. Higgs, Phys. Lett. 12 (1964) 132.

10. P.W. Higgs, Phys. Rev. Lett. 13 (1964) 508.

11. ALEPH, DELPHI, L3 and OPAL Collaborations, Phys. Lett. B 565 (2003) 61.

12. CDF Collaboration, D $\varnothing$ Collaboration, Tevatron New Physics, Higgs Working Group, arXiv:1207.0449 [hep-ex] (2012).

13. ALEPH, CDF, D $\varnothing$, DELPHI, L3, OPAL, SLD Collaborations, the LEP Electroweak Working, the Tevatron Electroweak Working Group, the SLD Electroweak and Heavy Flavour Groups, CERN-PH-EP-2010-095, arXiv:1012.2367 [hep-ex] (2010).

14. https://twiki.cern.ch/twiki/bin/view/LHCPhysics/CrossSections

15. ATLAS Collaboration, Phys. Rev. Lett. 108 (2012) 111803.

16. ATLAS Collaboration, Phys. Lett. B 710 (2012) 383.

17. ATLAS Collaboration, Phys. Lett. B 717 (2012) 70.

18. ATLAS Collaboration, Phys. Lett. B 717 (2012) 29.

19. ATLAS Collaboration, Phys. Lett. B 716 (2012) 62.

20. ATLAS Collaboration, Phys. Lett. B 718 (2012) 391.

21. ATLAS Collaboration, JHEP 09 (2012) 070.

22. ATLAS Collaboration, Phys. Lett. B 718 (2012) 369.

23. ATLAS Collaboration, ATLAS-CONF-2012-078, http://cds.cern.ch/record/1460390 .

24. ATLAS Collaboration, Phys. Rev. D 86 (2012) 032003. 
25. ATLAS Collaboration, ATLAS-CONF-2012-047, http://cds.cern.ch/record/1449796 .

26. ATLAS Collaboration, ATLAS-CONF-2012-092, http://cds.cern.ch/record/1460411 .

27. ATLAS Collaboration, ATLAS-CONF-2012-091, http://cds.cern.ch/record/1460410 .

28. ATLAS Collaboration, CMS Collaboration, http://cds.cern.ch/record/1379837 .

29. ATLAS Collaboration, ATLAS-CONF-2012-093, http://cds.cern.ch/record/1460439 .

30. ATLAS Collaboration, Phys. Lett. B 716 (2012) 1.

31. ATLAS Collaboration, Science 338 no. 6114 (2012) 1576.

32. CMS Collaboration, Phys. Lett. B 716 (2012) 30. 